\title{
ON THE PRESERVATION OF DIRECTION-CONVEXITY AND THE GOODMAN-SAFF CONJECTURE
}

\author{
Stephan Ruscheweyh and Luis C. Salinas
}

\begin{abstract}
Let $K(\varphi)$ be the set of univalent functions in the unit disk $\mathbf{D}$ which are convex in the direction $e^{i \varphi}$. We determine the set of analytic functions $g$ in $\mathbf{D}$ which preserve $K(\varphi)$ under the Hadamard product, i.e., $g * f \in K(\varphi)$ whenever $f \in K(\varphi)$. This result contains as a special case the proof of a conjecture of Goodman and Saff about $K(\varphi)$ and solves partially a multiplier problem concerning convex univalent harmonic functions in $\mathbf{D}$, posed by Clunie and Sheil-Small.
\end{abstract}

\section{Introduction}

A domain $M \subset \mathbf{C}$ is said to be convex in the direction $e^{i \varphi}$ if for every $a \in \mathbf{C}$ the set

$$
M \cap\left\{a+t e^{i \varphi}: t \in \mathbf{R}\right\}
$$

is either connected or empty. Let $K(\varphi)$ be the family of univalent analytic functions $f$ in the unit disk $\mathbf{D}$ with $f(\mathbf{D})$ convex in the direction $e^{i \varphi}$ and, similarly, $K_{H}(\varphi)$ with 'univalent analytic' replaced by 'univalent harmonic'. It is well-known (see W. Hengartner and G. Schober [5], A.W. Goodman and E.B. Saff [4]) that for $r_{0}:=\sqrt{2}-1<r<1$ generally $f \in K(\varphi)$ does not imply $f(r z) \in K(\varphi)$, but Goodman and Saff conjectured that such an implication may hold for $0<r \leq r_{0}$. Recently J. Brown [1] proved that

$$
f \in K(\varphi) \Rightarrow f(r \circ z) \in K(\psi), \quad \psi \in I(f),
$$

where $I(f) \subset[0,2 \pi)$ is a set of positive measure. It was not shown, however, that $\varphi \in I(f)$ and thus the conjecture remained open. We shall prove the following stronger result:

Theorem 1. Let $f \in K_{H}(\varphi), 0<r \leq r_{0}$. Then $f(r z) \in K_{H}(\varphi)$.

Research supported by the Fondo Nacional de Desarollo Científico y Tecnológico (FONDECYT, Grant 249/87), by the Universidad Técnica Federico Santa María (Grant 87.12.06), and by the German Academic Exchange Service (DAAD).

1980 Mathematics subject classification (1985 revision). Primary 30C45. 
This settles the Goodman-Saff conjecture even for univalent harmonic functions. In the analytic case, however, Theorem 1 is a very simple special case of the solution of the following multiplier problem ( $*$ denotes the Hadamard product):

Determine the set DCP of all analytic functions $g$ in $\mathbf{D}$ such that $g * f \in K(\varphi)$ for every $\varphi \in \mathbf{R}$ and every $f \in K(\varphi)$.

Theorem 2. Let $g$ be analytic in $\mathbf{D}$. Then $g \in \mathrm{DCP}$ if and only if

$$
\text { for each } \gamma \in \mathbf{R}: g+i \gamma z g^{\prime} \in K\left(\frac{\pi}{2}\right) \text {. }
$$

Theorem 1, for $f$ analytic, follows from Theorem 2 by choosing $g_{r}(z):=$ $1 /(1-r z)$ and showing that $g_{r} \in \mathrm{DCP}$ for $0<r \leq r_{0}$. If $f$ is harmonic in $\mathbf{D}, f=\overline{f_{1}}+f_{2}$ with $f_{1}, f_{2}$ analytic in $\mathbf{D}$ and $f_{1}(0)=0$, we may define for an analytic $g$

$$
f \tilde{*} g:=\overline{\left(f_{1} * g\right)}+\left(f_{2} * g\right) .
$$

It is not true that all functions $g$ satisfying $(1)$ preserve $K_{H}(\varphi)$ under the operation $\tilde{*}$ (Theorem 1, however, says that this is the case for $g_{r}$ ). For an example see Clunie and Sheil-Small [3, (5.21.1)] where the multiplier happens to satisfy (1). But out result does extend to the class $K_{H}$ of convex harmonic univalent functions $f$ (where 'convex' indicates that $f(\mathbf{D})$ is convex).

Theorem 3. Let $g$ be analytic in D. Then $f \tilde{*} g \in K_{H}$ for all $f \in K_{H}$ if and only if $g$ satisfies (1), i.e., $g \in \mathrm{DCP}$.

This theorem solves partially a problem of Clunie and Sheil-Small [3,(7.7)].

The members of $K(\varphi)$ are usually described analytically through a condition due to M.S. Robertson [7] (see also W.C. Royster and M. Ziegler [8]). Unfortunately, this condition is very difficult to deal with when it comes to convolutions (Hadamards products). In the proof of the basic Theorem 2 we shall use a completely different way, namely the concept of periodically monotone functions, introduced by I.J. Schoenberg [11].

Definition. Let $u$ be a real, continuous, $2 \pi$-periodic function. It is said to be periodically monotone $(u \in \mathrm{PM})$ if there exist numbers $\theta_{1}<\theta_{2}<\theta_{1}+2 \pi$ such that $u$ increases on $\left(\theta_{1}, \theta_{2}\right)$ and decreases on $\left(\theta_{2}, \theta_{1}+2 \pi\right)$.

We shall reduce the discussion of functions in DCP to the characterisation of certain integral kernels which preserve periodic monotonicity. And, as a result of this connection, we also obtain the following very handy criterion for $g$ to be in DCP.

Theorem 4. Let $g$ be non-constant and analytic in $\mathbf{D}$, continuous in $\overline{\mathbf{D}}$ with $u(\theta)=\operatorname{Re} g\left(e^{i \theta}\right)$ three times continuously differentiable. Then $g \in \mathrm{DCP}$ if and only if $u \in \mathrm{PM}$ with

$$
u^{\prime}(\theta) u^{\prime \prime \prime}(\theta) \leq\left(u^{\prime \prime}(\theta)\right)^{2}, \quad \theta \in \mathbf{R} .
$$




\section{Proofs}

Let $C_{2 \pi}^{k}$ denote the set of real $2 \pi$-periodic functions which are $k$ times continuously differentiable. For $u, v \in C_{2 \pi}^{0}$ we define

$$
(u * v):=\frac{1}{2 \pi} \int_{0}^{2 \pi} u(\psi) v(\theta-\psi) d \psi .
$$

There will be no confusion in using the same symbol $*$ for different convolutions since from the context it will be always clear which one is meant. In fact, there is a close connection between the two definitions: let $g, h$ be analytic in $\mathbf{D}$, continuous in $\overline{\mathbf{D}}, g(0)=0$, and set

$$
u(\theta):=\operatorname{Re} g\left(e^{i \theta}\right), \quad v(\theta):=\operatorname{Re} h\left(e^{i \theta}\right), \quad \theta \in \mathbf{R} .
$$

Then we have the important relation

$$
(u * v)(\theta)=\frac{1}{2} \operatorname{Re}(g * h)\left(e^{i \theta}\right), \quad \theta \in \mathbf{R} .
$$

(3) is readily verified by writing down the corresponding Fourier expansions.

A function $u \in C_{2 \pi}^{1}$ is said to preserve periodic monotonicity ( $u \in \mathrm{PMP}$ ) if

$$
u * v \in \mathrm{PM} \quad \text { for every } \mathrm{v} \in \mathrm{PM}
$$

Let $V_{n}$ be the de la Vallée-Poussin kernels:

$$
V_{n}(\theta):=\left(\begin{array}{c}
2 n \\
n
\end{array}\right)^{-1}(1+\cos \theta)^{n}, \quad \theta \in \mathbf{R}, n \in \mathbf{N}
$$

It is known (de la Vallée-Poussin [13]) that for $u \in C_{2 \pi}^{0}$ we have

$$
\lim _{n \rightarrow \infty}\left(V_{n} * u\right)(\theta)=u(\theta), \quad \theta \in \mathbf{R} .
$$

Furthermore, as has been shown by Pólya and Schoenberg [6], the $V_{n}$ are variation diminishing. These two properties imply: $n \in \mathbf{N}$.

Lemma 1. Let $u \in C_{2 \pi}^{0}$. Then $u \in \mathrm{PM}$ if and only if $V_{n} * u \in \mathrm{PM}$ for all

Similarly we obtain

Lemma 2. Let $u \in C_{2 \pi}^{1}$. Then $u \in \mathrm{PMP}$ if and only if $V_{n} * u \in \mathrm{PMP}$ for all $n \in \mathbf{N}$. 
Indeed, if $u \in \mathrm{PMP}, v \in \mathrm{PM}$ then, by Lemma $1, V_{n} * u \in \mathrm{PM}$ and hence $\left(V_{n} * v\right) * u=v *\left(V_{n} * u\right) \in \mathrm{PM}$, which implies $V_{n} * u \in \mathrm{PMP}$. In the other direction, if $v *\left(V_{n} * u\right) \in \mathrm{PM}$ for all $v \in \mathrm{PM}$ then, using dominated convergence,

$$
v * u=\lim _{n \rightarrow \infty}\left(V_{n} * v\right) * u \in \mathrm{PM}
$$

and hence $u \in$ PMP. The crucial part in the proof of Theorem 2 is contained in the following result.

Theorem 5. Let $u \in C_{2 \pi}^{1}$ be such that

$$
\tilde{u}(\theta):=u(\theta)-i u^{\prime}(\theta), \quad 0 \leq \theta \leq 2 \pi,
$$

is a (complex) Jordan curve with a convex interior domain. Then $u \in$ PMP.

We remark that a more general definition of the classes PM and PMP has been studied by Schoenberg [11], who also quotes a result of C. Loewner which says that (5) is essentially also a necessary condition for $u \in$ PMP. In another paper [10] we give the complete characterisation of the wider Schoenberg class. For our present purpose, however, this is of no relevance.

We shall reduce the proof of Theorem 5 to the following lemma which is of independent interest.

Lemma 3. Let $u$ be a trigonometric polynomial satisfying the assumptions of Theorem 5. Let $h \not \equiv$ const. be a function in $C_{2 \pi}^{0}$ such that $h$ has at most two sign changes in any interval of length $2 \pi$ and satisfies

$$
\frac{1}{2 \pi} \int_{0}^{2 \pi} h(\psi) d \psi=0
$$

Then $u * h$ has exactly two zeros (which are simple) in $[0,2 \pi)$.

Proof. We first note that $\tilde{u}$ is strongly convex, i.e., there are no three numbers $\theta_{1}<\theta_{2} \theta_{3}<\theta_{1}+2 \pi$ such that the points $\tilde{u}\left(\theta_{j}\right), j=1,2,3$, lie on a straight line. In fact, if they were, then by the convexity we conclude that $\tilde{u}(\theta)$ lies on that straight line, $\theta_{1} \leq \theta \leq \theta_{3}$. This gives a relation

$$
a u(\theta)+b u^{\prime}(\theta)+c=0
$$

on that interval, and since $u$ is a trigonometric polynomial, for all $\theta$. But then $\tilde{u}$ lies completely in that straight line, a contradiction to the assumption. We shall use this information in the following form: let $\psi_{1}<\psi_{2}<\psi_{1}+2 \pi$ and denote by $\operatorname{co}(A)$ the interior of the convex hull of a set $A \subset \mathbf{C}$. Then

$$
\operatorname{co}\left\{\tilde{u}(\psi): \psi_{1} \leq \psi_{\leq} \psi_{2}\right\} \cap \operatorname{co}\left\{\tilde{u}(\psi): \psi_{2} \leq \psi \leq \psi_{1}+2 \pi\right\}=\emptyset .
$$


Now let $\theta_{1}<\theta_{2}<\theta_{1}+2 \pi$ be such that

$$
h(\theta) \begin{cases}\geq 0, & \text { if } \theta_{1} \leq \theta \leq \theta_{2} \\ \leq 0, & \text { if } \theta_{2} \leq \theta \leq \theta_{1}+2 \pi\end{cases}
$$

Note that by (6) we can be sure that $h$ has at least two zeros in a period. We define the set $M=M\left(\theta_{1}, \theta_{2}\right)$ as the set of real $2 \pi$-periodic functions $g$, continuous in $I_{1}:=\left(\theta_{1}, \theta_{2}\right)$ and in $I_{2}=\left(\theta_{2}, \theta_{1}+2 \pi\right)$, such that

$$
g(\theta) \begin{cases}\geq 0, & \text { if } \theta \in I_{1}, \\ \leq 0, & \text { if } \theta \in I_{2},\end{cases}
$$

and

$$
1=\frac{1}{2 \pi} \int_{I_{1}} g(\psi) d \psi=-\frac{1}{2 \pi} \int_{I_{2}} g(\psi) d \psi
$$

Clearly $\varrho h \in M$ for some suitable $\varrho>0$. For $g \in M$ the function $v_{g}:=g * u$ is a trigonometric polynomial and we wish to show that this polynomial cannot have any multiple zero. In fact, for $\varrho \in \mathbf{R}$ we have

$$
v_{g}(\varphi)-i v_{g}^{\prime}(\varphi)=\frac{1}{2 \pi} \int_{I_{1}} g(\theta) \tilde{u}(\varphi-\theta) d \theta-\frac{1}{2 \pi} \int_{I_{2}}(-g(\theta)) \tilde{u}(\varphi-\theta) d \theta
$$

and from (9), (10) we conclude that

$$
\begin{gathered}
\frac{1}{2 \pi} \int_{I_{1}} g(\theta) \tilde{u}(\varphi-\theta) d \theta \in \operatorname{co}\left\{\tilde{u}(\psi): \varphi-\theta_{2} \leq \psi \leq \varphi-\theta_{1}\right\}, \\
\frac{1}{2 \pi} \int_{I_{2}}(-g(\theta)) \tilde{u}(\varphi-\theta) d \theta \in \operatorname{co}\left\{\tilde{u}(\psi): \varphi-\theta_{1}-2 \pi \leq \psi \leq \varphi-\theta_{2}\right\},
\end{gathered}
$$

and thus by ( 8 )

$$
v_{g}(\varphi)-i v_{g}^{\prime}(\varphi) \neq 0, \quad \varphi \in \mathbf{R} .
$$

Hence $v_{g}$ and $v_{g}^{\prime}$ can never vanish simultaneously and $v_{g}$ cannot have multiple zeros. Now assume that we can find at least one $g_{0} \in M$ such that $V_{g_{0}}$ has only two zeros (simple, of course) in a period. Then, if $v_{\varrho h}$ has more than two zeros in a period (but, because of the periodicity, an even number), then there exists a $\lambda \in(0,1)$ such that

$$
\lambda v_{g_{0}}+(1-\lambda) v_{\varrho h}=v_{\left[\lambda g_{0}+(1-\lambda) \varrho h\right]}
$$

has a double zero. But $M$ is a convex set and hence $\lambda g_{0}+(1-\lambda) \varrho h \in M$, a contradiction. 
What remains is to construct $g_{0}$. We set

$$
g_{0}(\theta)= \begin{cases}2 \pi /\left(\theta_{2}-\theta_{1}\right), & \text { if } \theta \in I_{1}, \\ 0, & \text { if } \theta=\theta_{1}, \theta_{2}, \\ -2 \pi /\left(\theta_{1}+2 \pi-\theta_{2}\right), & \text { if } \theta \in I_{2},\end{cases}
$$

and extend this definition periodically to $\mathbf{R}$. Then $g_{0} \in M$ and

$$
v_{g_{0}}(\varphi)=\frac{1}{\theta_{2}-\theta_{1}} \int_{I_{1}} u(\varphi-\theta) d \theta-\frac{1}{\theta_{1}+2 \pi-\theta_{2}} \int_{I_{2}} u(\varphi-\theta) d \theta
$$

and hence

$$
v_{g_{0}}^{\prime}=\left(\frac{1}{\theta_{2}-\theta_{1}}+\frac{1}{\theta_{1}+2 \pi-\theta_{2}}\right)\left(u\left(\varphi-\theta_{1}\right)-u\left(\varphi-\theta_{2}\right)\right) .
$$

The convexity of $\tilde{u}$ implies that $u \in \mathrm{PM}$ and since $u$ is a non-constant trigonometric polynomial $v_{g_{0}}^{\prime}$ has only two zeros in a period. The same is therefore true for $v_{g_{0}}$. Since $g_{0} \in M$ we conclude that $v_{g_{0}}$ has (exactly) two simple zeros in a period. This completes the proof of Lemma 3.

Proof of Theorem 5. It follows again from the variation diminishing property of the kernels $V_{n}$ and from

$$
V_{n} * u^{\prime}=\left(V_{n} * u\right)^{\prime}
$$

that $u_{n}:=V_{n} * u$ satisfies the assumptions of Theorem 5 . Using Lemma 2 we conclude that we have to prove Theorem 5 only for trigonometric polynomials $u$. Similarly, if $t * u \in \mathrm{PM}$ for all trigonometric polynomials $t \in \mathrm{PM}$, then $u \in \mathrm{PMP}$.

A non-constant trigonometric polynomial $t$ is in PM if and only if $t^{\prime}$ has exactly two sign changes in any period. Furthermore we obviously have

$$
\frac{1}{2 \pi} \int_{0}^{2 \pi} t^{\prime}(\psi) d \psi=0
$$

Hence, if $t \in \mathrm{PM}$, we can apply Lemma 3 to $h:=t^{\prime}$ and obtain that

$$
v^{\prime}=(t * u)^{\prime}=h * u
$$

has (exactly) two sign changes in a period. This proves $v \in \mathrm{PM}$ and hence $u \in$ PMP.

The geometric condition concerning $\tilde{u}$ in Theorem 5 can be replaced by a more analytic one if $u \in C_{2 \pi}^{3}$ : we can then describe the convexity by the monotonicity of the tangent rotation at $\tilde{u}$ and by ensuring that the total variation of the argument of the tangent vector is $2 \pi$. This leads immediately to: 
Lemma 4. Let $u \in C_{2 \pi}^{3}$ be non-constant and $\tilde{u}$ as in (5). Then $\tilde{u}$ fulfills the assumption of Theorem 5 if and only if $u \in \mathrm{PM}$ and

$$
u^{\prime}(\theta) u^{\prime \prime \prime}(\theta) \leq\left(u^{\prime}(\theta)\right)^{2}, \quad \theta \in \mathbf{R} .
$$

After these 'real' preliminaries we now turn to the discussion of $K(\varphi)$ and DCP. Also here we need a reduction to polynomial cases. We are working with the analytics version of the de la Vallée-Poussin kernels:

$$
W_{n}(z):=\left(\begin{array}{c}
2 n \\
n
\end{array}\right)^{-1} \sum_{k=0}^{n}\left(\begin{array}{c}
2 n \\
n+k
\end{array}\right) z^{k}, \quad z \in \mathbf{C}, n \in \mathbf{N} .
$$

Note that

$$
2 \operatorname{Re} W_{n}\left(e^{i \theta}\right)=V_{n}(\theta)+1, \quad \theta \in \mathbf{C}, n \in \mathbf{N} .
$$

Lemma 5. Let $g$ be analytic in D. Then $g \in K(\varphi)$ if and only if $W_{n} * g \in$ $K(\varphi)$ for $n \in \mathbf{N}$.

Proof. Without loss of generality we may assume $g(0)=0, \varphi=\frac{1}{2} \pi$. Let $g \in K(\pi / 2), \Gamma=g(\mathbf{D})$. We can construct a sequence of polygonal domains $\Gamma_{k}$ with

$$
0 \in \Gamma_{1} \subset \Gamma_{2} \subset \cdots \subset \Gamma, \quad \bigcup_{k \in \mathbf{N}} \Gamma_{k}=\Gamma,
$$

and $\Gamma_{k}$ convex in the direction of the imaginary axis. Let $g_{k}$ be the univalent functions in $\mathbf{D}$ with $g_{k}(0)=0, \arg g_{k}^{\prime}(0)=\arg ^{\prime}(0)$ and $g_{k}(\mathbf{D})=\Gamma_{\mathbf{k}}$. Then $g_{k} \in K(\pi / 2)$ and $g_{k} \rightarrow g$ locally uniformly in $\mathbf{D}$ by Caratheodory's kernel convergence. The functions $g_{k}$ extend continuously to $\partial \mathbf{D}$ and the directionconvexity is reflected by the property that $u_{k}(\theta):=\operatorname{Re} g_{k}\left(e^{i \theta}\right)$ is in PM. Hence, since $V_{n} \in \mathrm{PMP}$, we find using (3), (11), (12):

$$
\operatorname{Re}\left(W_{n} * g_{k}\right)=V_{n} * u_{k} \in \mathrm{PM} .
$$

The elements of $K(\pi / 2)$ are, in particular, close-to-convex univalent functions while the polynomials $W_{n}$ are convex univalent in $\mathbf{D}$ (Pólya and Schoenberg [6]). Hence, by the result of Ruscheweyh and Sheil-Small [9], we conclude that $W_{n} * g_{k}$ is close-to-convex univalent in $\mathbf{D}$. This fact together with (13) implies that $W_{n} * g_{k} \in K(\pi / 2)$. But obviously $W_{n} * g_{k} \rightarrow W_{n} * g$ locally uniformly in $\mathbf{D}$ and hence $W_{n} * g \in K(\pi / 2)$ for $n \in \mathbf{N}$.

If, on the other hand, $W_{n} * g \in K(\pi / 2)$ for $n \in \mathbf{N}$ then we have $g \in K(\pi / 2)$ since $W_{n} * g \rightarrow g$ locally uniformly in $\mathbf{D}$.

Lemma 6. Let $g$ be analytic in $\mathbf{D}$. Then $g \in \mathrm{DCP}$ if and only if $W_{n} * g \in$ DCP for $n \in \mathbf{N}$. 
Proof. Lemma 5 shows, in particular, that $W_{n} \in \mathrm{DCP}$ and since DCP is obviously closed under convolutions (i.e., $f, g \in \mathrm{DCP}$ implies $f * g \in \mathrm{DCP}$ ) we have $W_{n} * g \in \mathrm{DCP}$ if $g \in \mathrm{DCP}$. If $W_{n} * g \in \mathrm{DCP}$ for $n \in \mathbf{N}$ then for $f \in K(\varphi)$ :

$$
g *\left(W_{n} * f\right)=\left(W_{n} * g\right) * f \in K(\varphi) .
$$

With $n \rightarrow \infty$ we obtain $g * f \in K(\varphi)$ and thus $g \in \mathrm{DCP}$.

For the proof of Theorem 2 we shall need one further result, due to Clunie and Sheil-Small [3]:

Lemma 7. Let $f_{1}, f_{2}$ be analytic in $\mathbf{D}, f_{1}(0)=0$. Then $F=\overline{f_{1}}+f_{2} \in K_{H}$ if and only if

$$
f_{2}-e^{i \varphi} f_{1} \in K\left(\frac{\varphi}{2}\right), \quad \varphi \in \mathbf{R} .
$$

Proof of Theorem 2. We show first that (1) is necessary for $g$ to be in DCP. We have $g+i \gamma z g^{\prime}=g * f_{\gamma}$ where

$$
f_{\gamma}(z)=\frac{1}{1-z}+i \gamma \frac{z}{(1-z)^{2}}, \quad \gamma \in \mathbf{R} .
$$

These functions are close-to-convex univalent and map $\mathbf{D}$ onto $\mathbf{C}$ minus a vertical slit. Thus they are in $K(\pi / 2)$ and (1) turns out to be a special case of the directionconvexity preservation of $g$.

Now let $g$ satisfy (1). We observe that this implies that $g$ is convex univalent in $\mathbf{D}$. In fact, since $g * f_{\gamma} \in K(\pi / 2)$ we see that

$$
\left(g * f_{\gamma}\right)^{\prime}(0)=g^{\prime}(0) \cdot f_{\gamma}^{\prime}(0) \neq 0
$$

and thus $g^{\prime}(0) \neq 0$. Furthermore, for $z \in \mathbf{D}$,

$$
0 \neq\left(g * f_{\gamma}\right)^{\prime}(z)=\frac{1}{z}\left(z g^{\prime} * f_{\gamma}\right)=g^{\prime}+i \gamma\left(z g^{\prime}\right)^{\prime}
$$

and hence

$$
\frac{z g^{\prime \prime}(z)}{g^{\prime}(z)}+1 \neq \frac{i}{\gamma}, \quad \gamma \in \mathbf{R}, \quad z \in \mathbf{D}
$$

which gives

$$
\operatorname{Re}\left(\frac{z g^{\prime \prime}(z)}{g^{\prime}(z)}+1\right)>0, \quad z \in \mathbf{D}
$$

the convexity condition for $g$.

The convexity of $g$ implies [9] that $f * g$ is univalent for $f$ close-to-convex, in particular for $f \in K(\varphi)$. 
We found already that $W_{n} \in \mathrm{DCP}, n \in \mathbf{N}$, and therefore

$$
W_{n} *\left(g+i \gamma z g^{\prime}\right)=\left(W_{n} * g\right)+i z \gamma\left(W_{n} * g\right)^{\prime} \in K\left(\frac{1}{2} \pi\right), \quad \gamma \in \mathbf{R},
$$

which shows that $W_{n} * g$ satisfies (1) as well. In view of Lemma 6 this implies that we have to prove the sufficiency part of Theorem 2 only for polynomials $g$. Similarly, using Lemma 5 , we see that we have to prove $f * g \in K(\varphi)$ only for polynomials $f \in K(\varphi)$. Obviously we may restrict ourselves again to the case $\varphi=\pi / 2$, and we may assume $g(0)=0$. We know already that $f * g$ is univalent in $\mathbf{D}$. Hence to prove $f * g \in K(\pi / 2)$ we just have to prove that

$$
\operatorname{Re}\left[(f * g)\left(e^{i \theta}\right)\right]=2\left(\operatorname{Re} f\left(e^{i \theta}\right)\right) *\left(\operatorname{Re} g\left(e^{i \theta}\right)\right) \in \mathrm{PM}
$$

under the assumption that $\operatorname{Re} f\left(e^{i \theta}\right) \in \mathrm{PM}$. But this is surely true if we can show that $u(\theta):=\operatorname{Re} g\left(e^{i \theta}\right) \in \mathrm{PMP}$.

We rewrite (1) as follows: let $i \gamma=\left(1+e^{i \varphi}\right) /\left(1-e^{i \varphi}\right), 0<\varphi<2 \pi$, and note that

$$
\arg \left[i\left(1-e^{i \varphi}\right)\right]=\frac{1}{2} \varphi, \quad 0<\varphi 2 \pi .
$$

Hence

$$
\left(1-e^{i \varphi}\right)\left(g+i \gamma z g^{\prime}\right)=\left(g+z g^{\prime}\right)-e^{i \varphi}\left(g-z g^{\prime}\right) \in K(\varphi / 2),
$$

for $0<\varphi<2 \pi$. The limiting case $\gamma \rightarrow \infty$ can be used to show that (15) holds for $\varphi=0$ as well. We now apply Lemma 7 and deduce that

$$
F(z):=\overline{g-z g^{\prime}}+g+z g^{\prime}=2\left(\operatorname{Re} g(z)+i \operatorname{Im} z g^{\prime}(z)\right) \in K_{H} .
$$

This clearly implies that

$$
\frac{1}{2} F\left(e^{i \theta}\right)=u(\theta)-i u^{\prime}(\theta), \quad 0 \leq \theta<2 \pi,
$$

is a convex curve in the sense of Theorem 5: $u$ belongs to PMP, and this completes the proof of Theorem 2.

We note that the last steps in this proof are invertible: if the curve (17) is convex in the sense of Theorem 5, then, by a Theorem of Choquet [2], the statement (16) also holds true. Using the other direction of Lemma 7 we conclude that the function $g$ satisfies (1). We have shown:

Lemma 8. Let $g$ be analytic in $\mathbf{D}$, continuous in $\overline{\mathbf{D}}$ with $u(\theta)=\operatorname{Re} g\left(e^{i \theta}\right) \in$ $C_{2 \pi}^{1}$. Then $g \in \mathrm{DCP}$ if and only if $u$ fulfills the assumptions of Theorem 5 . 
The assertion of Theorem 4 is just a combination of Lemma 4 and Lemma 8 .

Proof of Theorem 1. Using $g_{r}(z):=1 /(1-r z)$ we obtain

$$
u_{r}(\theta)=\operatorname{Re} g_{r}\left(e^{i \theta}\right)=\frac{1}{2}+\frac{1}{2} \frac{1-r^{2}}{1+r^{2}-2 r \cos \varphi} .
$$

It is a matter of straightforward calculus to show that $u_{r}(\theta)$ satisfies the conditions of Theorem 4 for $0<r \leq r_{0}$. Theorem 1 follows for $f \in K(\varphi)$. A result of Clunie and Sheil-Small [3, Theorem 5.3] extends this immediately to $K_{H}(\varphi)$.

Proof of Theorem 3. That $f \tilde{*} g \in K_{H}$ for $f \in K_{H}$ and $g \in \mathrm{DCP}$ follows from Theorem 2 and Lemma 7. On the other hand, Clunie and Sheil-Small [3, (5.5.4)] have shown that

$$
f_{0}(z)=\overline{\frac{1}{1-z}-\frac{z}{(1-z)^{2}}}+\frac{1}{1-z}+\frac{z}{(1-z)^{2}} \in K_{H} .
$$

Hence, if $g$ preserves harmonic convexity, we must have $F=f_{0} \tilde{*} g \in K_{H}$ where $F$ is exactly the function (16). As we have seen in the deduction of Lemma 8 this is equivalent to the fact that $g$ satisfies (1) and hence to $g \in \mathrm{DCP}$. 


\section{References}

[1] Brown, J.E.: Level sets for functions convex in one direction. - Proc. Amer. Math. Soc. $100,1987,442-446$.

[2] Choquet, G.: Sur un type de transformation analytique generalisant la representation conforme et definie au moyen de fonctions harmoniques. - Bull. Sci. Math. (2) 69, $1945,156-165$.

[3] Clunie, J.G., and T. Sheil-Small: Harmonic univalent functions. - Ann. Acad. Sci. Fenn. Ser. A I Math. 9, 1984, 3-25.

[4] Goodman, A.W., and E.B. SafF: On univalent functions convex in one direction. - Proc. Amer. Math. Soc. 73, 1979, 183-187.

[5] Hengartner, W., and G. Schober: A remark on level curves for domains convex in one direction. - Applicable Anal. 3, 1973, 101-106.

[6] Pólya, G., and I.J. Schoenberg: Remarks on the de la Vallée- Poussin means and convex conformal maps of the circle. - Pacific J. Math. 8, 1958, 295-334.

[7] Robertson, M.S.: Analytic functions starlike in one direction. - Amer. J. Math. 58, 1936, $465-472$.

[8] Royster. W.C., and M. Ziegler: Univalent function convex in one direction. - Publ. Math. Debrecen 23, 1976, 339-345.

[9] Ruscheweyh, St., and T. Sheil-Small: Hadamard products of schlicht functions and the Polya-Schoenberg conjecture. - Comment. Math. Helv. 48, 1973, 119-135.

[10] Ruscheweyh, St., and L.C. Salinas: On the preservation of periodic monitonicity. Preprint, 1987.

[11] Schoenberg, I.J.: On variation diminishing approximation methods. - Numerical approximation, edited by R. Langer. University of Wisconsin Press, 1959, 249-274.

[12] DE la VAllée-Poussin, Ch.J.: Sur l'approximation des fonctions d'une variable reelle et de leurs derivees par des polynomes et des suites limites de Fourier. - Bull. Acad. Roy. Belgique (Classe de sciences) 3, 1908, 193-254.

Universidad Técnica Federico Santa María

Departamento de Matemática

Valparaíso

Chile

Received 3 November 1987
Universität Würzburg

Mathematisches Institut

D-8700 Würzburg

Federal Republic of Germany 